\title{
Adolescentes y patrones de género: consumo televisivo y su seguimiento en redes sociales
}

Adolescents and gender patterns: television consumption and social media sharing

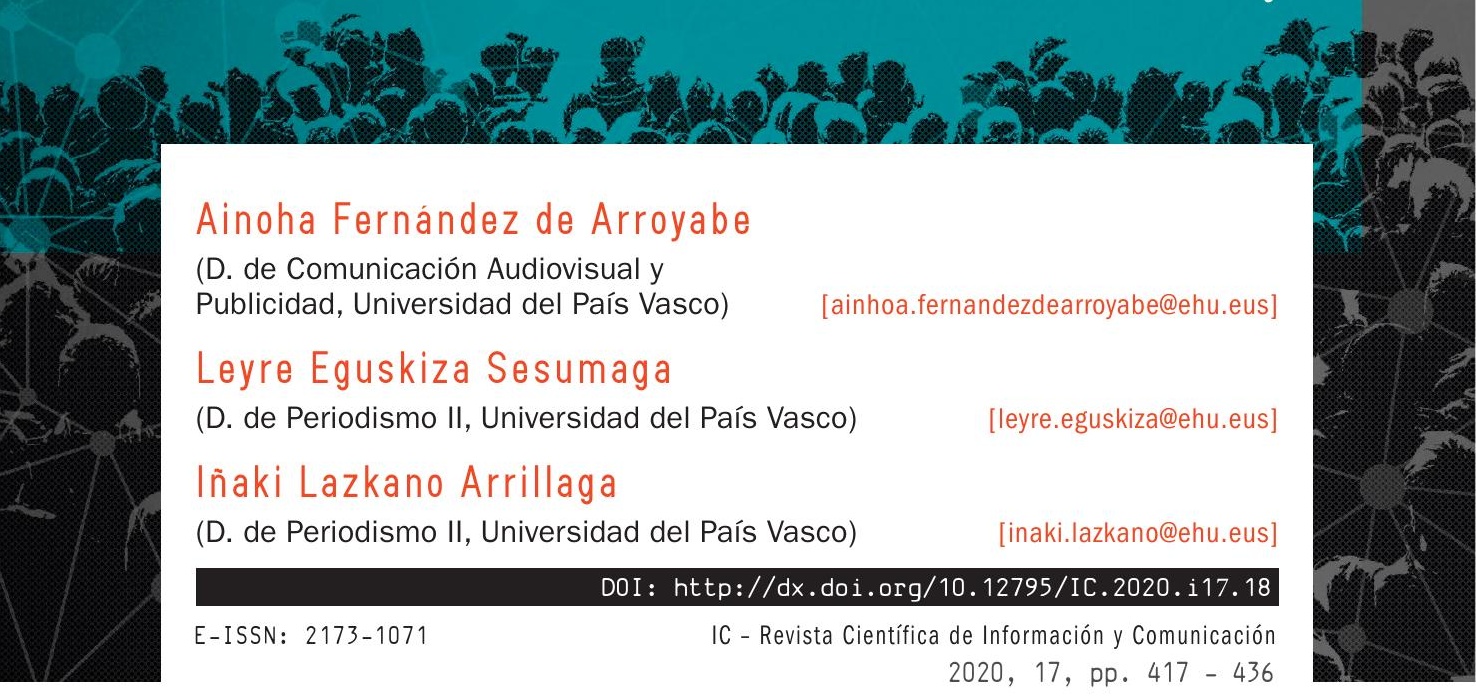

\section{Resumen}

Este artículo analiza los patrones de consumo televisivo de los adolescentes vascos y su seguimiento en redes sociales y WhatsApp, atendiendo a variables de género y edad. El sondeo cuantitativo realizado a estudiantes de Educación Secundaria de Guipúzcoa muestra que la televisión se mantiene como medio de referencia entre los jóvenes, quienes perpetúan y amplifican los patrones de consumo masculinos y femeninos tradicionales en el seguimiento de sus contenidos en redes.

\section{Abstract}

This research analyses the television consumption patterns of Basque adolescents and the way they follow these products up through social media and WhatsApp, according to their gender and age. The quantitative survey filled out by secondary education students of Guipúzcoa shows that television continues to be seen as the main media among teenagers. In addition, male and female traditional patterns are not only consolidated but also amplified through their social media habits.

Palabras clave

Adolescentes, género, programa de televisión, medios sociales, estereotipos. 


\section{Keywords}

Adolescents, gender, television programmes, social media, stereotypes.

\section{Sumario}

1. Introducción

2. Marco teórico

2.1. Una generación conectada: del televisor al consumo multipantalla

2.2. Patrones y estereotipos de género en el consumo televisivo adolescente

3. Metodología

4. Análisis de los resultados

4.1. Patrones de consumo televisivo y género: compartimentos estancos

4.1.1. Películas: cine romántico para ellas y acción para ellos

4.1.2. Teleseries: un terreno de éxito adolescente

4.1.3. Comedias: el humor triunfa entre los jóvenes

4.1.4. Telerrealidad: un universo femenino

4.1.5. Deportes: un espacio masculino

4.2. Televisión social: seguimiento de contenidos en redes y mensajería digital

4.3. Consumo y seguimiento de contenidos televisivos por género

4.3.1. Perfil de consumo femenino: temas y valores

4.3.2. Perfil de consumo masculino: temas y valores

5. Discusión y conclusiones

6. Bibliografía

\section{Summary}

1. Introduction

2. Theoretical framework

2.1. Net Generation: From television to multiscreen consumption

2.2. Gender patterns and stereotypes in the adolescents' television consumption

3. Methods

4. Analysis and results

4.1. Television consumption patterns and gender: watertight compartment

4.1.1. Movies: romantic films for girls and action for boys

4.1.2. TV series: a field of teen success

4.1.3. Comedies: humour, the favourite of adolescents

4.1.4. Reality shows: a female universe

4.1.5. Sport: a male land

4.2. Social television: content following-up through social media and instant messaging

4.3. Television content consumption and following-up by gender

\subsubsection{Female consumption profile: themes and values}

4.3.2. Male consumption profile: themes and values

5. Discussion and conclusions

6. References 


\section{Introducción}

La actual generación de adolescentes ha crecido en paralelo al continuo desarrollo de la tecnología, redes sociales y dispositivos móviles. En este contexto hiperconectado han desarrollado un paradigma propio de consumo de contenidos audiovisuales, en el que la televisión continúa ocupando una posición hegemónica como medio principal de socialización. Los adolescentes consumen televisión y lo hacen mayoritariamente con su progenie, aunque han incorporado una nueva práctica que modifica el sentido del visionado y que ha sido bautizada como televisión social: el seguimiento de programas con sus pares mediante las redes o servicios de mensajería como WhatsApp.

Las distintas investigaciones realizadas hasta la fecha constatan la existencia de patrones de consumo de televisión -ficción, series, comedias, programas, telerrealidad y deportes- diametralmente opuestos en función del género. El objetivo de este trabajo es analizar la incidencia de esta nueva práctica, es decir, el seguimiento e intercambio de opiniones sobre los programas que han visto en televisión a través de las redes, en dichos patrones de consumo entre los adolescentes vascos en función del género y el curso (edad). De esta manera, la investigación plantea las siguientes Research Questions:

RQ1: ¿Cuáles son los patrones de consumo de contenidos de televisión de los chicos y chicas vascos/as?

RQ2: ¿Cuáles son las producciones más populares y comentadas a través de las redes y WhatsApp entre los jóvenes vascos/as?

RQ3: ¿Cómo incide el mencionado fenómeno de la televisión social en los patrones de consumo audiovisual de la juventud vasca?

\section{Marco teórico}

\subsection{Una generación conectada: del televisor al consumo multipantalla}

La nueva generación de nativos digitales, conocida como Net Generation (Fernández-Planells \& Figueras, 2014), ha dado lugar a unos jóvenes "multitarea, conectados, prosumidores, sociales y móviles" que hacen de las herramientas digitales los 
recursos esenciales para sus actividades de ocio, relacionadas éstas principalmente con la socialización y el entretenimiento (Viñals, Abad \& Aguilar, 2014: 53). Así, su tiempo libre se erige ahora en torno a la personalización, el consumo a demanda y la multiplicidad de pantallas, dando lugar a un modelo “móvil, transmedia y multisoporte” (Viñals, 2016: 99).

Las nuevas vías de acceso a los medios, sin embargo, no han logrado variar los cinco usos tradicionales atribuidos al consumo mediático adolescente distinguidos por Jeffrey Jensen Arnett (1995): entretenimiento, formación de identidad, estimulación de sensaciones, confrontación de emociones negativas e identificación con una cultura propia (p. 521).

En la búsqueda de una o varias de estas motivaciones, se despliega ante ellos el gran abanico de posibilidades del mundo digital, no exento de peligros que los convierten en un grupo poblacional más vulnerable. Una elevada (y en ocasiones involuntaria) exposición de su imagen, el ciberacoso o la dependencia de Internet son problemáticas sobre las que se ha venido alertando en los últimos años (Livingstone \& Helsper, 2010; Catalina, López de Ayala \& García, 2014).

Sin perder de vista estos riesgos, y en consonancia con el entorno digital en el que crecen, los adolescentes se han establecido como "un público diferenciado con usos y hábitos mediáticos característicos” (Fernández-Planells \& Figueras, 2014: 89). En este contexto, el smartphone se erige como dispositivo estrella (Sádaba \& Vidales, 2015; Mascheroni \& Ólafsson, 2016), haciendo de la ubicuidad y portabilidad sus principales ventajas (Viñals, 2016: 101). Se convierte, pues, en un elemento "multiuso e interactivo", lejos de su concepción como mero dispositivo de comunicación (Méndiz, De Aguilera \& Borges, 2011:78).

Los medios de comunicación tradicionales también han dejado paso a nuevas plataformas audiovisuales como YouTube, a herramientas de socialización como las redes sociales y a productos interactivos de ocio como los videojuegos (Gewerc, Fraga \& Rodés, 2017). La incursión de estos recursos digitales no posee, al menos de momento, un carácter excluyente con respecto al modelo convencional, sino que la modificación de la dieta mediática ha traído consigo una diversificación de los roles atribuidos a cada uno de ellos (Marín, Yaguana \& Barrazueta, 2013). En otras palabras, a pesar de la disminución de su consumo entre la juventud, la televisión es considerada un modo de desconexión mientras que Internet se asocia al entretenimiento y la socialización. Este nuevo escenario ha propiciado, a su vez, una diversificación del entorno al que vinculan cada pantalla. Los dispositivos de mayor tamaño como el televisor son destinados a un entorno familiar y los más pequeños (smartphones o tabletas) a un consumo más individualizado (Teso \& Piñuel, 2014: 2). 
Así pues, la televisión mantiene su monopolio como el medio con mayor implantación en los hogares (García-Matilla \& Molina, 2008), aunque su evolución natural deriva hacia una smart tv que camina más allá de las parrillas de programación secuenciales y que posibilita a los jóvenes interactuar con otros semejantes (Teso \& Piñuel, 2014: 14). De hecho, dicho sector poblacional ha dejado atrás el modelo de audiencia pasiva y se define como "prosumidora", creando sus propios contenidos audiovisuales (García-Matilla \& Molina, 2008: 89).

Estas nuevas formas de consumo de televisión e Internet (hipertelevisión) se basan en la multiplicidad de formatos y pantallas (González-Aldea \& Medina-de-laViña, 2014: 4), con lo que va tomando fuerza el visionado multipantalla y multitarea, simultaneando diferentes actividades y dispositivos (Méndiz, De Aguilera \& Borges, 2011:78). Entre ellos destaca la popularmente denominada segunda pantalla, es decir, el consumo paralelo de televisión en pantalla tradicional y de Internet en el smartphone.

\subsection{Patrones y estereotipos de género en el consumo televisivo adolescente}

Lo mencionado hasta el momento permite contextualizar el actual escenario global en el que convergen jóvenes y televisión, donde persisten las diferencias de género en lo que respecta a los hábitos de consumo audiovisual y donde algunos de los productos más populares continúan perpetuando estereotipos y roles de género (Pibernat, 2017; Masanet \& Fedele, 2019).

La televisión se presenta como un medio de referencia para el ocio del público joven, a pesar del descenso experimentado en los últimos años. Así lo corrobora el informe estatal Análisis televisivo 2019 (Barlovento Comunicación), que sitúa en más de una hora y media diaria la franja de consumo entre los 13 y los 24 años. De estos trabajos se desprenden cuestiones relevantes en torno a las motivaciones y contenidos favoritos en lo que respecta al visionado televisivo. A grandes rasgos, la ficción, los deportes y la comedia son los contenidos predilectos de esta generación (Medrano \& Cortés; 2008; López-Vidales, González-Aldea \& Medina-de-la-Viña, 2014; Masanet, 2016).

Un análisis detallado por géneros, sin embargo, pone de manifiesto dos patrones de consumo enfrentados y diferenciados entre sí, tal y como se aprecia en sendos estudios previos (Adriaens, Van-Damme \& Courtois, 2011; Alcolea, 2014). Las chicas prefieren los programas basados en las relaciones interpersonales como telerrealidad, programas del 
corazón o series de ficción. Los chicos, por su parte, consumen en mayor medida los contenidos vinculados a los deportes o al humor (Medrano \& Cortés; 2008; Medrano, Aierbe \& Orejudo, 2009; Masanet, 2016).

Los valores trasladados por estos productos audiovisuales también han sido objeto de numerosas investigaciones (Rodríguez-Martínez, Medrano, Aierbe \& Martínez-deMorentin, 2013; Cuervo, Medrano \& Aierbe, 2016; Korres \& Elexpuru, 2017), analizando en profundidad variables como el género, edad o contexto cultural. De la misma manera, el mundo académico ha estudiado con especial atención la representación de género en series y películas, los productos audiovisuales que gozan de mayor popularidad en este sector poblacional (Fedele \& García-Muñoz, 2010; Álvarez, González-de-Garay \& Frutos, 2015).

Junto a la aparición de nuevas plataformas de visionado, las redes sociales han modificado la socialización de los más jóvenes en lo que a consumo televisivo se refiere. $Y$ es que gracias a la convergencia entre medios convencionales y nuevas tecnologías se dan nuevas formas de consumo que han dejado "obsoleto" el paradigma tradicional (Teso \& Piñuel, 2014). Esta televisión social se cimienta sobre el consumo de contenidos a través de las redes sociales, utilizadas cada vez más por los adolescentes para acceder a la actualidad informativa (Casero, 2012; García, Tur-Viñes \& Pastor, 2019), pero también para la interacción con los emisores y entre la propia audiencia (Teso \& Piñuel, 2014: 3-4).

De hecho, hoy día resulta incuestionable la penetración mayoritaria de las redes sociales entre el público más joven (Livingstone, 2008; Nuñez, García, \& Hermida, 2012; García, López-de-Atala \& Catalina, 2013). La misma tendencia queda reflejada en el informe elaborado por el Observatorio Vasco de Juventud, donde queda patente que la totalidad de la juventud vasca es usuaria habitual de alguna red social (2019: 18). El servicio de mensajería más utilizado para el intercambio de contenidos es WhatsApp, con un $99 \%$ de usuarios, seguido de Youtube (99\%), Instagram (81\%) y Facebook (58\%).

La socialización, entendida como el establecimiento de nuevas relaciones afectivas y el intercambio de experiencias, es la motivación común que impulsa a los jóvenes a la utilización de estas plataformas (Colás, González \& De-Pablos, 2013). Sádaba $\&$ Vidales (2015) apostillan que "en la gestión que los adolescentes hacen de las redes sociales está el inicio de la construcción de su propio capital social” (p. 78), es decir, el cúmulo de recursos adquiridos mediante las relaciones interpersonales.

Una vez más, sin embargo, tras esas motivaciones comunes subyacen comportamientos diferenciados por géneros que se traducen en una mayor presencia femenina en las redes sociales, donde tienen un papel más activo y una mayor exposición (Espinar \& González, 2009; Sánchez \& De Frutos, 2012). Asimismo, los chicos les confieren 
una utilización más instrumental, centrada en el refuerzo de su propia identidad, mientras que las chicas las emplean como modo de expresión de sus sentimientos y pensamientos (Estébanez \& Vázquez, 2013; Alonso, Rodríguez, Lameiras \& Carrera, 2015).

\section{Metodología}

La investigación se basa un sondeo cuantitativo realizado a 2.426 adolescentes -de 12 a 16 años- pertenecientes a los cuatro cursos de Educación Secundaria Obligatoria en Guipúzcoa (País Vasco) ${ }^{1}$. El método utilizado ha sido el muestreo por conglomerados, estratificado por afijación proporcional que parte de la distribución geográfica de los centros y los niveles educativos, y cuya unidad es el grupo. La muestra es una selección aleatoria de 60 centros y un total de 120 grupos, 30 por cada curso. El error muestral máximo es de $+/-1,90 \%$ y el nivel de confianza estadístico es del 95\% (en el supuesto más desfavorable de $p=q=0,5)$.

Este trabajo se vincula a una investigación de mayor extensión sobre la influencia de la tecnología y la presencia del euskera -lengua cooficial del territorio- en los hábitos de consumo, creación y difusión de contenidos audiovisuales del público adolescente. El artículo presenta datos inéditos sobre el consumo televisivo -ficción, series, comedias, programas, telerrealidad y deportes-, así como sobre el seguimiento y socialización de estos contenidos en redes sociales entre los jóvenes vascos, en función de las variables de género y curso (edad). Es por esto que el diseño del cuestionario incluye una selección de los títulos de las teleseries, comedias y programas de telerrealidad universales o de formato global más vistos y emitidos durante la realización del estudio -diciembre 2016/enero 2017-, tanto en cadenas estatales públicas y privadas, como en la televisión autonómica vasca (EITB). Asimismo, en cada categoría se ha incorporado una pregunta abierta para identificar los títulos más seguidos a través de las redes y WhatsApp.

El análisis cuantitativo y cualitativo de los resultados obtenidos ha permitido definir los patrones de consumo televisivo de los jóvenes en el contexto de la televisión social, dando respuesta a las preguntas de investigación planteadas inicialmente.

1 Aunque el estudio se haya realizado en este territorio, los resultados son extrapolables por el tamaño del universo, 28.817 jóvenes repartidos en 108 centros, la heterogeneidad socioeconómica del mismo y la universalidad de los contenidos sometidos a estudio. 


\section{Análisis de los resultados}

\subsection{Patrones de consumo televisivo y género: compartimentos estancos}

\subsubsection{Películas: cine romántico para ellas y acción para ellos}

El consumo de géneros cinematográficos de los chicos y chicas vascos resulta por completo diferente y se caracteriza por la marcada oposición entre los contenidos preferidos por unos y otras. Sí es común el interés de los jóvenes por estos productos audiovisuales, ya que el porcentaje de quienes afirman no ver películas es mínimo $(3,1 \%)$ y son más chicos $(3,6)$ que chicas $(1,9)$.

TABLA 1

Distribución del género cinematográfico de las peliculas en función del género

\begin{tabular}{|c|c|c|c|c|c|c|c|}
\hline PELICULAS & Total & Chicos & Chicas & PELICULAS & Total & Chicos & Chicas \\
\hline Comedia & $77,9 \%$ & $76,4 \%$ & 79,5 & Terror & $47,8 \%$ & $48,1 \%$ & $47,4 \%$ \\
\hline Aventura & $72,3 \%$ & $75,3 \%$ & $69,4 \%$ & Romántico & $42,2 \%$ & $14,7 \%$ & $69 \%$ \\
\hline Acción & $69,9 \%$ & $80 \%$ & $60,1 \%$ & Histórico & $24,1 \%$ & $30 \%$ & $18,3 \%$ \\
\hline Policiaca & $59,5 \%$ & $65 \%$ & $54,2 \%$ & Documental & $14,9 \%$ & $19,7 \%$ & $10,2 \%$ \\
\hline Ciencia ficcion & $53,8 \%$ & $62,6 \%$ & $45,2 \%$ & Porno & $6,6 \%$ & $11,6 \%$ & $1,6 \%$ \\
\hline
\end{tabular}

Fuente: Elaboración propia.

La diferencia significativa que presentan las películas románticas entre las mujeres evidencia que se trata de un género netamente femenino. Igualmente, las películas de acción, policíacas, de ciencia ficción, históricas y, en particular, el porno son contenidos claramente masculinos, puesto que la presencia de chicos se acentúa fuertemente entre sus consumidores. Cabe subrayar el peso específico que también ellos también tienen en el porno y que apunta al interés por el sexo inherente a los chicos.

\subsubsection{Teleseries: un terreno de éxito adolescente}

Las series son otro de los contenidos predilectos de los adolescentes vascos; de hecho, solo el 2,7\% afirma no verlas. Tal y como ocurriera con el cine, y a pesar de que el 
consumo de teleseries es similar entre chicos y chicas, sí se aprecian algunas diferencias significativas detalladas a continuación.

TABLA 2

Distribución de las series de televisión más vistas en función del género

\begin{tabular}{|c|c|c|c|c|c|c|c|}
\hline SERIES & Total & Chicos & Chicas & SERIES & Total & Chicos & Chicas \\
\hline Hawai 5.0 & $65,3 \%$ & $67,6 \%$ & $63,2 \%$ & El principe & $13,6 \%$ & $9,5 \%$ & $17,4 \%$ \\
\hline Castle & $51,4 \%$ & $43,8 \%$ & $59 \%$ & El cuerpo del delito & $13 \%$ & $7,2 \%$ & $18,7 \%$ \\
\hline Mentes criminales & $45,8 \%$ & $41,5 \%$ & $49,9 \%$ & Águila roja & $11 \%$ & $11,9 \%$ & $10,1 \%$ \\
\hline CSI & $44,4 \%$ & $42,5 \%$ & $46,2 \%$ & Narcos & $10,1 \%$ & $14,5 \%$ & $5,9 \%$ \\
\hline NCSI: Los Ángeles & $38,6 \%$ & $38,8 \%$ & $38,5 \%$ & Pequeñas mentirosas & $9,8 \%$ & $2,2 \%$ & $17,1 \%$ \\
\hline The Walking Dead & $19,8 \%$ & $25,7 \%$ & $14,2 \%$ & Crónicas vampiricas & $6,8 \%$ & $3,8 \%$ & $9,7 \%$ \\
\hline El mentalista & $15,2 \%$ & $13,5 \%$ & $16,9 \%$ & Stranger Things & $4,7 \%$ & 4,2 & $5,1 \%$ \\
\hline Juego de Tronos & $14.3 \%$ & $14,3 \%$ & $15,4 \%$ & Mr Robot & $2,8 \%$ & 3,9 & $1,8 \%$ \\
\hline
\end{tabular}

Fuente: Elaboración propia.

En línea con la tendencia anterior, las series femeninas por antonomasia son Castle y El cuerpo del delito, ambas policíacas, las cuales además ocupan el segundo y sexto lugar entre las favoritas. Las series eminentemente masculinas, por su parte, son The Walking Dead y Narcos, aunque ambas se sitúan en posiciones algo más discretas de la lista.

\subsubsection{Comedias: el humor triunfa entre los jóvenes}

En la línea con la tendencia anterior, el consumo de comedias es similar entre géneros, aunque los y las adolescentes muestran algunas preferencias diferencias. Siguiendo la tendencia dominante, las comedias netamente femeninas son las que presentan tramas románticas: el musical Go!azen y Alli abajo, que ocupan la cuarta y sexta posición entre las más vistas, y la minoritaria Jane the Virgin. Por el contrario, los chicos se imponen en comedias de humor ácido como Los Simpson, que se posiciona la tercera de mayor consumo. y The Big Bang Theory y Vaya semanita $(34,6 \%)$, son la séptima y octava, respectivamente. 
TABLA 3

Distribución de las comedias más vistas en función del género

\begin{tabular}{|c|c|c|c|c|c|c|c|}
\hline COMEDIAS & Total & Chicos & Chicas & COMEDIAS & Total & Chicos & Chicas \\
\hline La que se avecina & $68,3 \%$ & $71,3 \%$ & $65,5 \%$ & Alli abajo & $35,3 \%$ & $30,4 \%$ & $40,2 \%$ \\
\hline Gym Tony & $61,7 \%$ & $63,5 \%$ & $60,1 \%$ & Vaya semanita & $34,6 \%$ & $40,9 \%$ & $28,4 \%$ \\
\hline Los Simpson & $58,2 \%$ & $69,7 \%$ & $47,2 \%$ & Dos hombres y medio & $30,1 \%$ & $33,2 \%$ & $27,3 \%$ \\
\hline Golazen & $42,4 \%$ & $27,2 \%$ & $57,2 \%$ & Como conoci a & $23,8 \%$ & $23,8 \%$ & $23,9 \%$ \\
\hline The Big Bang Theory & $41,4 \%$ & $46,4 \%$ & $36,6 \%$ & Jane the Virgin & $4,4 \%$ & $1,8 \%$ & $7 \%$ \\
\hline Modern Family & $39,3 \%$ & $36,8 \%$ & $42 \%$ & Merli & $3,1 \%$ & $6,2 \%$ & $3,6 \%$ \\
\hline
\end{tabular}

Fuente: Elaboración propia.

\subsubsection{Telerrealidad: un universo femenino}

Salvo el reality show de aventura extrema El conquistador del fin del mundo, una producción de la cadena autonómica vasca EITB que gusta tanto a chicos como a chicas, el resto de formatos de telerrealidad y programas del corazón presentan notables diferencias en función del género. Una vez más, queda evidenciada la hegemonía de la audiencia femenina en este terreno, al tiempo que se subraya el desinterés de los chicos por este tipo de contenidos.

TABLA 4

Distribución de los programas de telerrealidad más vistos en función del género

\begin{tabular}{|c|c|c|c|c|c|c|c|}
\hline TELERREALIDAD & Total & Chicos & Chicas & TELERREALIDAD & Total & Chicos & Chicas \\
\hline First Dates & $44,6 \%$ & $36,8 \%$ & $52,2 \%$ & Quiero ser & $18,1 \%$ & $4,9 \%$ & $31 \%$ \\
\hline Cámbiame & $40,7 \%$ & $19,7 \%$ & $61,4 \%$ & Tú al norte y yo al sur & $10,7 \%$ & $8,4 \%$ & $13 \%$ \\
\hline $\begin{array}{c}\text { Mujeres y hombres } \\
\text { y viceversa }\end{array}$ & $35 \%$ & $22,6 \%$ & $47 \%$ & Sálvame & $10,6 \%$ & $7,9 \%$ & $13,2 \%$ \\
\hline $\begin{array}{l}\text { El conquistador del } \\
\text { fin del mundo }\end{array}$ & $27,7 \%$ & $26,6 \%$ & $28,9 \%$ & $\begin{array}{c}\text { Granjero busca } \\
\text { esposa }\end{array}$ & $10,4 \%$ & $8 \%$ & $12,7 \%$ \\
\hline
\end{tabular}




\begin{tabular}{|c|c|c|c|c|c|c|c|}
\hline TELERREALIDAD & Total & Chicos & Chicas & TELERREALIDAD & Total & Chicos & Chicas \\
\hline Gran Hermano & $27,6 \%$ & $17,8 \%$ & $37,2 \%$ & Sálvame Deluxe & $9,6 \%$ & $7 \%$ & $12 \%$ \\
\hline Gu ta Gutarrak & $25,6 \%$ & $18,7 \%$ & $32,4 \%$ & No sabe, no contesta & $23,4 \%$ & $35,3 \%$ & $11,7 \%$ \\
\hline$-0 .-1 \%$ &
\end{tabular}

Fuente: Elaboración propia.

El programa de belleza Cámbiame es una excepción coyuntural y los programas del corazón como Sálvame no son tan vistos, lo que habla de que los formatos de telerrealidad que pivotan sobre las relaciones amorosas entre hombres y mujeres como First Dates, Mujeres y hombres y viceversa, Gran Hermano y la producción de EITB Gu ta Gutarrak son los preferidos por las chicas. A este respecto, señalar que la audiencia de estos programas crece con la edad. El alumnado de segundo ciclo de Educación Secundaria es el que más sigue Mujeres y hombres y viceversa $\left(39,8 \%\right.$ en $3^{\circ}$ curso y $40,6 \%$ en $\left.4^{\circ}\right)$; mientras que la audiencia más alta de Gran Hermano (34\%) y Quiero ser (23,6\%) se da en tercer curso.

\subsubsection{Deportes: un espacio masculino}

Algo contrario de lo que ocurre con los programas de telerrealidad, se puede afirmar que el deporte es un contenido netamente masculino, de acuerdo a las marcadas diferencias de género registradas en todas las categorías.

TABLA 5

Distribución de los deportes en función del género

\begin{tabular}{|c|c|c|c|c|c|c|c|}
\hline DEPORTE & Total & Chicos & Chicas & DEPORTE & Total & Chicos & Chicas \\
\hline Fútbol & $65,3 \%$ & $76 \%$ & $54,8 \%$ & Tenis & $21,5 \%$ & $29,6 \%$ & $13,7 \%$ \\
\hline Baloncesto & $35,8 \%$ & $46,6 \%$ & $25,4 \%$ & Ciclismo & $19,9 \%$ & $27,9 \%$ & $12,1 \%$ \\
\hline Fórmula 1 & $27,1 \%$ & $40,6 \%$ & $14,1 \%$ & Boxeo & $15,9 \%$ & $25,4 \%$ & $6,6 \%$ \\
\hline Motociclismo & $25,8 \%$ & $41,1 \%$ & $10,9 \%$ & Traineras & $14,5 \%$ & $18,2 \%$ & $10,8 \%$ \\
\hline Pelota vasca & $24,8 \%$ & $32,8 \%$ & $17 \%$ & Deportes de riesgo & $13,3 \%$ & $21,2 \%$ & $5,6 \%$ \\
\hline Balonmano & $24,2 \%$ & $28,6 \%$ & $19,9 \%$ & Deporte rural vasco & $8,1 \%$ & $11,3 \%$ & $5,1 \%$ \\
\hline Atletismo & $23,4 \%$ & $29,6 \%$ & $17,3 \%$ & Monte & $7,5 \%$ & $10,1 \%$ & $5,1 \%$ \\
\hline
\end{tabular}

Fuente: Elaboración propia. 
Indiscutiblemente, el fútbol es el deporte rey, ya que mantiene una distancia de treinta puntos con la siguiente categoría, el baloncesto, tras la que se alinea una heterogénea lista de deportes que tienen arraigo entre los chicos vascos. La apatía de las féminas respecto al deporte queda patente en la afirmación "no veo deportes" (\%23,6 chicas y $9,6 \%$ chicos), a la que hay que sumar la desafección respecto a los deportes violentos como el boxeo o los deportes de riesgo, el monte y el deporte rural vasco.

\subsection{Televisión social: seguimiento de contenidos en redes y mensajería digital}

El seguimiento y socialización de los contenidos televisivos consumidos por los adolescentes en redes y servicios de mensajería se confirma como una tendencia mayoritaria, ya que solo un $6 \%$ es ajeno a esta actividad. WhatsApp es el medio más utilizado para ello: el $63,4 \%$ trata estos programas con otra persona y el $54,7 \%$ lo hace en grupos de contenidos temáticos, mientras que el 47,2\% prefiere la conversación interpersonal. De otro lado, Instagram es la red social más usada con este fin $(44,2 \%)$, y tanto Twitter como Facebook son minoritarias (6\% ambas).

Los contenidos televisivos son el tema de conversación favorito de los jóvenes por delante de los videojuegos (43,4\%), que ocupan el último lugar en sus hábitos. De hecho, las películas $(81,8 \%)$, teleseries $(65,4 \%)$, telerrealidad $(47 \%)$ y deporte $(46,2 \%)$ son los temas principales. Por géneros, las chicas conversan más sobre películas $(87,5 \%)$, series $(73,3 \%)$ y telerrealidad $(63,1 \%)$, mientras que los chicos lo hacen en mayor medida sobre videojuegos $(73,5 \%)$ y deporte $(65,3 \%)$. Además, como ya se ha mencionado, a medida que cumplen años siguen más los reality show (39,4\% en primer curso y $55 \%$ en cuarto).

La pregunta abierta en torno a las películas que han visto y comentado en redes sociales sirve para dibujar nítidas diferencias de género: las chicas nombran más Un monstruo viene a verme (10\%) y la película romántica 3 metros sobre el cielo $(8,1 \%)$, basada en la novela de Federico Moccia; los chicos, por su parte, las sagas Star Wars (5,1\%), Fast and Furious (4,3\%) y Assassin's Creed (4,2\%).

En cuanto a la comedia, Go!azen $(18,6 \%)$ es la más comentada, mientras que el resto se sitúa a gran distancia: Hawaii 5.0. (8,5\%), Castle (4\%), The Walking Dead (2,7\%), The Big Bang Theory (2,6\%) y Juego de Tronos (2\%), Pequeñas mentirosas (1,9\%), Teen Wolf $(1,8 \%)$ y Los Simpson (1,3\%). Las chicas hablan más sobre Go!azen (27,3\%), Castle $(5,7 \%)$, Pequeñas mentirosas $(3,2 \%)$ y Teen Wolf $(3,1 \%)$; los chicos, de Hawaii $5.0 .(12 \%)$ y The Walking Dead (4,7\%). 
Por último, respecto a los programas de telerrealidad como Gran Hermano (9,9\%) y Mujeres y hombres y viceversa (7,8\%), ambos emitidos por Telecinco, junto con Gu ta gutarrak (7,1\%), de EITB, son el tema estrella de conversación. Hay que subrayar que, a pesar de estar entre sus contenidos favoritos, los adolescentes apenas hablan sobre First Dates $(1,1 \%)$ o Cámbiame (1\%). Gran Hermano (17,3\%), Mujeres y hombres y viceversa $(13,8 \%)$ y Gu ta gutarrak (12\%) son los programas más comentados por las chicas y por la audiencia de mayor edad (Gran Hermano con el 13,3\% y Mujeres y hombres y viceversa con el 11,9\%).

\subsection{Consumo y seguimiento de contenidos televisivos por género}

La tabla que se presenta a continuación constituye una pormenorizada radiografía de los patrones de consumo y seguimiento de los contenidos televisivos más vistos/comentados por los adolescentes vascos de ambos géneros, así como de los temas principales sobre los que conversan.

TABLA 6

Patrones de consumo de televisión y seguimiento en redes sociales en función del género

\section{PERFIL DE CONSUMO FEMENINO}

\begin{tabular}{|c|c|c|c|}
\hline CONTENIDO & Género / titulo lista cerrada & $\begin{array}{l}\text { Titulos seguimiento } \\
\text { redes o WhatsApp }\end{array}$ & Temas \\
\hline PELÍCULAS & Románticas & $\begin{array}{c}\text { Un monstruo viene a } \\
\text { verme y } 3 \text { metros sobre } \\
\text { el cielo }\end{array}$ & \multirow{5}{*}{$\begin{array}{c}\text { Trama romántica: } \\
\text { adultos, amor } \\
\text { adolescente } \\
\text { Amor madre-hijo } \\
\text { Mujeres protagonistas } \\
\text { Relaciones de pareja }\end{array}$} \\
\hline SERIES & $\begin{array}{c}\text { Castle, El cuerpo del delito, } \\
\text { Pequeñas mentirosas }\end{array}$ & Go!azen, Castle y & \\
\hline COMEDIAS & $\begin{array}{c}\text { Go!azen, Alli abajo y Jane } \\
\text { the Virgin }\end{array}$ & Pequeñas mentirosas & \\
\hline TELERREALIDAD & $\begin{array}{l}\text { First Dates, Mujeres y } \\
\text { hombres y viceversa, Gran } \\
\text { Hermano, Gu ta gutarrak } \\
\text { (EITB), Sálvame y Granjero } \\
\text { busca esposa. }\end{array}$ & $\begin{array}{c}\text { Gran Hermano, Mujeres y } \\
\text { hombres y viceversa, Gu } \\
\text { ta gutarrak }\end{array}$ & \\
\hline DEPORTES & No veo deportes & No hay conversaciones & \\
\hline
\end{tabular}




\section{PERFIL DE CONSUMO MASCULINO}

\begin{tabular}{|c|c|c|c|}
\hline CONTENIDO & Género / titulo lista cerrada & $\begin{array}{l}\text { Titulos seguimiento } \\
\text { redes o WhatsApp }\end{array}$ & Temas \\
\hline PELICULAS & $\begin{array}{l}\text { Acción, policiacas, ciencia } \\
\text { ficcion, históricas y porno. }\end{array}$ & $\begin{array}{l}\text { Star Wars, Fast and } \\
\text { Furious y Assassin's Creed }\end{array}$ & \multirow{5}{*}{$\begin{array}{c}\text { Violencia } \\
\text { Policiacas y de terror } \\
\text { Comedias ácidas } \\
\text { Porno } \\
\text { Deporte }\end{array}$} \\
\hline SERIES & The Walking Dead y Narcos & \multirow{2}{*}{$\begin{array}{l}\text { La que se avecina, Hawaii } \\
\text { 5.0., The Walking Dead y } \\
\text { Los Simpson }\end{array}$} & \\
\hline COMEDIAS & $\begin{array}{l}\text { Los Simpson, The Big Bang } \\
\text { Theory y Vaya semanita }\end{array}$ & & \\
\hline TELERREALIDAD & $\begin{array}{l}\text { Desinterés: *en todo caso, } \\
\text { First Dates y Mujeres y } \\
\text { hombres y viceversa }\end{array}$ & $\begin{array}{l}\text { No hay titulos destacados } \\
\text { entre los chicos }\end{array}$ & \\
\hline DEPORTES & $\begin{array}{l}\text { Futbol, baloncesto, } \\
\text { motociclismo y pelota }\end{array}$ & Deporte & \\
\hline
\end{tabular}

Fuente: Elaboración propia.

Tal y como muestran los datos expuestos hasta el momento, existe una estrecha correlación entre la tipología y los títulos de los programas que más ven y siguen los chicos y chicas, ratificando así la pervivencia de patrones de consumo diferenciados por géneros, y en consecuencia, de temas y valores que no favorecen la igualdad dentro de este contexto de televisión social.

\subsubsection{Perfil de consumo femenino: temas y valores}

La trama romántica y el protagonismo femenino son las claves de la ficción -películas, series y comedia- que consumen y siguen las féminas, en la que también se abordan las relaciones de amistad entre chicas y entre ambos géneros. El liderazgo femenino en entornos profesionales masculinos es minoritario y, al mismo tiempo, el único factor que compensa la visión dominante de estas ficciones en las que de forma constante se proyectan modelos normativos tanto en lo que respecta a las relaciones amorosas amor romántico- como de amistad entre chicas -rivalidad- 0 entre chicos y chicas -lealtad femenina-, así como al rol de cuidadoras de las jóvenes en la familia.

La telerrealidad es un terreno femenino por excelencia y, sobre todo, aquellos programas que exhiben con frivolidad el flirteo entre adolescentes de ambos géneros $y$ cuyo visionado aumenta notablemente con la edad. Estos contenidos plantean una 
relación desequilibrada que enfatiza la sumisión y cosificación de las chicas, es decir, un modelo contrario a la igualdad de género. Por último, el desinterés absoluto por el deporte puede deberse a que un gran número de las competiciones emitidas por televisión son de equipos masculinos.

\subsubsection{Perfil de consumo masculino: temas y valores}

Los contenidos consumidos y compartidos por el público masculino en redes sociales dibujan un perfil algo más heterogéneo que el de las chicas. En la ficción películas, series y comedias-, de un lado, el denominador común de la acción, la aventura, el entramado policíaco y el terror es la violencia; de otro, la ironía caustica es el de las comedias y, por último, el porno evidencia el gran interés de los chicos por el sexo explícito.

La ironía es un valor positivo que contrasta con la normalización de la violencia y, sobre todo, del sexo explícito. El porno es el referente de la educación sexual de los chicos y su modelo se basa en la satisfacción del deseo masculino y la sumisión de las mujeres. Este aspecto resulta perjudicial a la hora de promover relaciones igualitarias entre chicos y chicas. A este respecto hay que señalar que, a pesar de que no consuman demasiada telerrealidad, los programas más populares entre ellos son los que exhiben relaciones desiguales entre hombres y mujeres. En esto coinciden con las adolescentes, una circunstancia que alerta sobre el efecto nocivo que causan estos populares contenidos.

Por último, el deporte es el territorio propio de los chicos, donde se constata la hegemonía de las competiciones masculinas. Las chicas parecen no tener aun cabida en un terreno que exalta la superioridad de la fuerza física de los hombres.

\section{Discusión y conclusiones}

Transitando por la senda que marcan las investigaciones previas en el área, este estudio pone en valor la popularidad de la llamada televisión social, subrayando la relación directa existente entre ver la televisión y chatear o conversar en WhatsApp, lo que implica una transformación en el sentido del visionado. El ciclo de vida del contenido va más allá y se establece un vínculo de afinidad entre sus seguidores adolescentes. Esto es algo que ya sucedía antes, la novedad es que ahora este vínculo es virtual, recoge las impresiones de unos y otros por escrito y se canaliza principalmente a través de WhatsApp, tanto en conversaciones interpersonales como en grupos temáticos. Dicho factor otorga un valor 
añadido a los contenidos que despiertan el suficiente interés para ser merecedores de generar una corriente más o menos estable de comentarios entre los menores. Todo apunta a que las conversaciones en WhatsApp y, en menor medida, en Instagram podrían ser consideradas como el germen del fandom, fenómeno fan que toma cuerpo en páginas web y otros formatos digitales en torno a los que se crea una comunidad global seguidora de un contenido.

En este contexto, el argumento central del trabajo resulta de especial relevancia, ya que corrobora la existencia de patrones de consumo femenino y masculino muy definidos y opuestos entre sí, que no solo se mantienen en el consumo televisivo, sino que se refuerzan en las conversaciones de las redes sociales y plataformas de mensajería instantánea. En otras palabras, la evolución tecnológica parece no haber incidido en los hábitos de consumo televisivo masculinos y femeninos.

Así, la pervivencia de los patrones de género es compleja y poliédrica. Los creadores y programadores de contenidos repiten las fórmulas de éxito, aunque estas vayan en contra de los valores de igualdad de género en los que la sociedad se esfuerza en educar a los jóvenes. No existe una regulación sobre los contenidos y la autorregulación en la industria audiovisual choca con las prioridades del negocio. Solo la presión de la opinión pública puede hacer que un contenido de este tipo se retire de la programación de cadenas públicas, como fue el caso del programa vasco de telerrealidad Gu ta gutarrak (EITB).

El género es una construcción cultural que se vehicula, entre otros agentes socializadores, a través de la televisión y que se amplifica en los nuevos medios, por consiguiente, ¿es posible cambiar los estereotipos audiovisuales de género?, ¿es posible triunfar entre los adolescentes con contenidos acordes a la igualdad?, ¿cuál es el papel de las instituciones o de los progenitores? Estas son solo algunas incógnitas a las que deberían responder las futuras investigaciones acerca de esta cuestión.

\section{Bibliografía}

Adriaens, F., Van-Damme, E. \& Courtois, C. (2011). The spatial and social contexts of television-viewing adolescents. Poetics, 39(3), 205-227. https:// doi. org/10.1016/j.poetic.2011.03.001

Alcolea, G. (2014). Análisis del consumo adolescente, con variables de género, de series y videojuegos: formas de acceso y actividad multitarea. VI Congreso Internacional Latina de Comunicación Social, 1-14. Tenerife: Universidad de la Laguna. Recuperado de https://bit.ly/380gxpt 
Alonso, P., Rodríguez, Y., Lameiras, M. \& Carrera, M. V. (2015). Hábitos de uso en las Redes Sociales de los y las adolescentes: análisis de género. Revista de Estudios e Investigación en Psicología y Educación, 13, 54-57. https://doi.org/10.17979/ reipe.2015.0.13.317

Álvarez, C., González-de-Garay, B. \& Frutos, F. J. (2015). Representación de género. Las películas españolas contemporáneas de adolescentes (2009-2014). Revista Latina de Comunicación Social, 70, 934-960. https://doi.org/10.4185/RLCS-2015-1079

Arnett, J. J. (1995). Adolescents' uses of media for self-socialization. Journal of Youth and Adolescence, 24(5), 519-533. https:// doi.org/10.1007/BF01537054

Barlovento Comunicación (2019). Análisis televisivo 2019. Madrid: Barlovento Comunicación. Recuperado de https://bit.ly/2NADyNE

Casero, A. (2012). Más allá de los diarios: el consumo de noticias de los jóvenes en la era digital. Comunicar, 20(39), 151-158. https://doi.org/10.3916/C392012-03-05

Catalina, B., López-de-Ayala, M. C. \& García, A. (2014). Los riesgos de los adolescentes en Internet: los menores como actores y víctimas de los peligros de Internet. Revista Latina de Comunicación Social, 69, 462-485. https://doi. org/10.4185/RLCS-2014-1020

Colás, P., González,T. \& De-Pablos, J. (2013). Juventud y redes sociales: Motivaciones y usos preferentes. Comunicar, 20(40), 5-23. https://doi.org/10.3916/C402013-02-01

Cuervo, S. L., Medrano, M. \& Aierbe, A. (2016). Values perceived by adolescents on television: cross-cultural and gender differences. Educación XX1, 19(2), 383-404. Recuperado de https://bit.ly/2smdpdW

Espinar, E. \& González, M. J. (2009). Jóvenes en las redes sociales virtuales: un análisis exploratorio de las diferencias de géneros. Feminimo/s, 14, 87-106. Recuperado de https://bit.ly/2FMs8C5

Estébanez, I. \& Vázquez, N. (2013). La desigualdad de género y el sexismo en las redes sociales. Una aproximación cualitativa al uso que hacen de las redes sociales las y los jóvenes. Vitoria-Gasteiz: Servicio central de publicaciones del Gobierno Vasco. Recuperado de https://bit.ly/2RdcncE 
Fedele, M. \& García-Muñoz, N. (2010). El consumo adolescente de la ficción seriada. Vivat Academia, 111, 47-64. https:// doi.org/10.15178/va.2010.111.47-64

Fernández-Planells. A. \& Figueras. M. (2014). De la guerra de pantallas a la sinergia entre pantallas: el multitasking en jóvenes. En Huertas, A. \& Figueras, M. (Ed.) Audiencias juveniles y cultura digital (pp. 87-105). Barcelona: Institut de la Comunicació, Universitat Autònoma de Barcelona. Recuperado de https://bit.ly/2RbY48e

García, A., López-de-Ayala, M. C. \& Catalina, B. (2013). Hábitos de uso en Internet y en las redes sociales de los adolescentes españoles. Comunicar, 21(41), 195-204. https:// doi.org/10.3916/C41-2013-19

García, A., Tur-Viñes, V. \& Pastor, Y. (2018). Consumo mediático de adolescentes y jóvenes. Noticias, contenidos audiovisuales y medición de audiencias. ICONO 14, 16(1), 22-46. https://doi.org/10.7195/ri14.v16i1.1101

García-Matilla, A. \& Molina, J. P. (2008). Televisión y jóvenes en España. Comunicar, 16(31), 83-90. https://doi.org/10.3916/c31-2008-01-0010

Gewerc, A., Fraga, F. \& Rodés, V. (2017). Niños y adolescentes frente a la Competencia Digital. Entre el teléfono móvil, youtubers y videojuegos. Revista Interuniversitaria de Formación del Profesorado, 89, 171-186. Recuperado de https://bit.ly/3a57jj7

Korres, 0. \& Elexpuru, I. (2017). Análisis de los valores percibidos por los adolescentes en el medio televisivo. Valores percibidos por adolescentes en la TV. Infancia y Aprendizaje: Journal for the Study of Education and Development, 40(4), 782-811. https://doi.org/1 $0.1080 / 02103702.2017 .1370821$

Livingstone, S. (2008). Taking Risky Opportunities in Youthful Content Creation: Teenagers' Use of Social Networking Sites for Intimacy, Privacy and Self-expression. New Media \& Society, 10(3), 393-411. https://doi. org/10.1177/1461444808089415

Livingstone, S. \& Helsper, E. (2010). Balancing Opportunities and Risks in Teenagers' Use of the Internet: The Role of Online Skills and Internet self-efficacy. New Media \& Society, 12(2), 309-329. https://doi.org/10.1177/1461444809342697 
López-Vidales, N., González-Aldea, P. \& Medina-de-la-Viña, E. (2014). Contenidos televisivos y jóvenes en la era digital. II Congreso Internacional Latina de Comunicación Social, 1-20. Tenerife: Universidad de la Laguna. Recuperado de https:// bit.ly/3aaytoM

Marín, I., Yaguana, H. A. \& Barrazueta, P. (2013). Estudio sobre el uso y hábitos de consumo de medios de comunicación de la adolescencia en Loja (Ecuador). $V$ Congreso Internacional Latina de Comunicación Social, 1-13. Tenerife: Universidad de la Laguna. Recuperado de https://bit.ly/36TdYem

Masanet, M. J. (2016). Pervivencia de los estereotipos de género en los hábitos de consumo mediático de los adolescentes: Drama para las chicas y humor para los chicos. Cuadernos. info, 39, 39-53. https://doi.org/10.7764/cdi.39.1027

Masanet, M. J. \& Fedele, M. (2019). El "chico malote" y la "chica responsable": modelos aspiracionales y representaciones juveniles en las teen series españolas. Palabra Clave, 22(2), 1-27. https://doi.org/10.5294/pacla.2019.22.2.5

Mascheroni, G. \& Ólafsson, K. (2016). The Mobile Internet: Access, Use, Opportunities and Divides among European Children. New Media \& Society, 18(8), 1657-1679. https://doi.org/10.1177/1461444814567986

Medrano, C. \& Cortés, A. (2008). ¿Persisten los estereotipos sociales en la dieta televisiva de los adolescentes? Comunicar, 16(31), 381-386. https://doi. org/10.3916/c31-2008-03-022

Medrano, C., Aierbe, A. \& Orejudo, S. (2009). El perfil de consumo televisivo en adolescentes: diferencias en función del sexo y estereotipos sociales. Infancia y Aprendizaje: Journal for the Study of Education and Development, 32(3), 293-306. https://doi.org/10.1174/021037009788964150

Méndiz, A., De-Aguilera, M. \& Borges, E. (2011). Actitudes y valoraciones de los jóvenes ante la TV móvil. Comunicar, 18(36), 77-85. https://doi.org/10.3916/ C36-2011-02-08

Núñez, P., García, M. L. \& Hermida, L. A. (2012). Tendencias de las relaciones sociales e interpersonales de los nativos digitales y jóvenes en la web 2.0. Revista Latina de Comunicación Social, 67, 179-204. https://doi.org/10.4185/RLCS067-952-179-206 


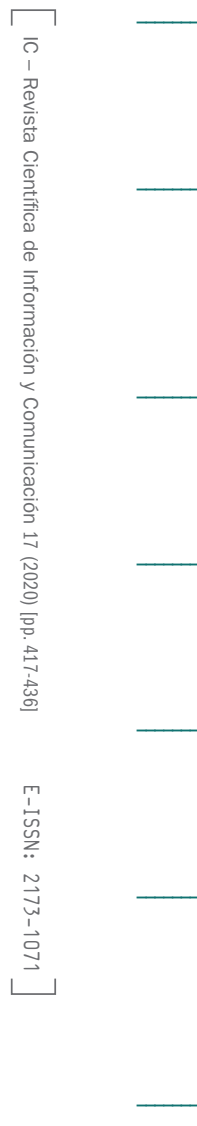

Observatorio Vasco de la Juventud (2019). La juventud vasca en las redes sociales. Vitoria-Gasteiz: Departamento de Educación, Política Lingüística y Cultura, Gobierno Vasco. Recuperado de https://bit.ly/37290vH

Pibernat, M. (2017). ¿Nuevas socializaciones, viejas cuestiones? Adolescencia y género en la era audiovisual. Investigaciones feministas: papeles de estudios de mujeres, feministas y de género, 8(2), 529-544. https://doi.org/10.5209/ INFE.54976

Rodríguez-Martínez, A., Medrano, C., Aierbe, A. \& Martínez-de-Morentin, J. I. (2013). Television Viewing Habits and the Values Perceived by Adolescents: A Cross-cultural Study. Revista de Educación, 361, 513-538. Recuperado de https://bit.ly/2R1w317

Sádaba, C. \& Vidales, M. J. (2015). El impacto de la comunicación mediada por la tecnología en el capital social: adolescentes y teléfonos móviles. Virtualis, 11(1), 75-92. Recuperado de https://bit.ly/2RkfMGy

Sánchez, M. \& De-Frutos, B. (2012). Adolescentes en las redes sociales ¿marca el género la diferencia. TELOS: Cuadernos de Comunicación e Innovación, 92, 1-9. Recuperado de https://bit.ly/2ReqIWq

Teso, G. \& Piñuel, J. L. (2014). Multitarea, Multipantalla y Práctica social del consumo de Medios entre los jóvenes de 16 a 29 años en España. VI Congreso Internacional Latina de Comunicación Social, 1-15. Tenerife: Universidad de la Laguna. Recuperado de https://bit.ly/3a96IT1

Viñals, A., Abad, M. \& Aguilar, E. (2014). Jóvenes conectados: Una aproximación al ocio digital de los jóvenes españoles. Communication Papers, 3(4), 52-68. Recuperado de https://bit.ly/2NqypYh

Viñals, A. (2016). El ocio conectado, móvil, transmedia y multisoporte de los jóvenes en la era digital. Journal of Communication, 13, 99-113. https://doi. org/10.14201/fjc20161399113 